\title{
GLOBAL DYNAMICS OF STATIONARY SOLUTIONS OF THE EXTENDED FISHER-KOLMOGOROV EQUATION
}

\author{
JAUME LLIBRE ${ }^{1}$, MARCELO MESSIAS $^{2}$ AND PAULO R. DA SILVA ${ }^{3}$
}

\begin{abstract}
In this paper we study the fourth order differential equation $\frac{d^{4} u}{d t^{4}}+q \frac{d^{2} u}{d t^{2}}+u^{3}-u=0$, which arises from the study of stationary solutions of the Extended Fisher-Kolmogorov equation. Denoting $x=$ $u, y=\frac{d u}{d t}, z=\frac{d^{2} u}{d t^{2}}, v=\frac{d^{3} u}{d t^{3}}$ this equation becomes equivalent to the polynomial system $\dot{x}=y, \quad \dot{y}=z, \quad \dot{z}=v, \quad \dot{v}=x-q z-x^{3}$ with $(x, y, z, v) \in \mathbb{R}^{4}$ and $q \in \mathbb{R}$. As usual, the dot denotes derivative with respect to the time $t$. Since the system has a first integral we can reduce our analysis to a family of systems on $\mathbb{R}^{3}$. We provide the global phase portrait of these systems in the Poincaré ball (i.e. in the compactification of $\mathbb{R}^{3}$ with the sphere $\mathbb{S}^{2}$ of the infinity).
\end{abstract}

\section{Introduction And Statement of main RESUlts}

The classical equations of mathematical physics are typically linear second order differential equations. However, many problems in the sciences and in engineering are intrinsically nonlinear. The Fisher-Kolmogorov equation

$$
\frac{\partial u}{\partial t}=\frac{\partial^{2} u}{\partial x^{2}}+u-u^{3}
$$

was originally proposed in 1937 to model the interaction of dispersal and fitness in biological populations. The EFK-equation or more precisely the extended Fisher-Kolmogorov equation,

$$
\frac{\partial u}{\partial t}=-\gamma \frac{\partial^{4} u}{\partial x^{4}}+\frac{\partial^{2} u}{\partial x^{2}}+u-u^{3}, \quad \gamma>0
$$

was proposed in 1988 as a higher order model equation for physical systems that are bistable (i.e the EFK-equation have two uniform states $u(x)=$ \pm 1 which are stable, separated by a third uniform state $u(x)=0)$. For

2010 Mathematics Subject Classification. 37G15,37D45.

Key words and phrases. Integrability, Extended Fisher-Kolmogorov equation, Poincaré compactification, global dynamics.

* The first author is partially supported by the MICIIN/FEDER grant MTM200803437, the Generalitat de Catalunya grant 2009SGR-410 and ICREA Academia. The second author is supported by CNPq-Brazil under the project 305204/2009-2. The third author is partially supported by CNPq and FAPESP. All the authors are supported by the Int. Coop. Proj. CAPES/MECD-TQED II and PHB-2009-0025. 
stationary solutions, that is the solutions which do not depend on the time $t$, the EFK-equation reduces to the ordinary differential equation

$$
-\gamma \frac{d^{4} u}{d x^{4}}+\frac{d^{2} u}{d x^{2}}+u-u^{3}=0, \quad \gamma>0 .
$$

By the transformation

$$
x=\sqrt[4]{\gamma} \bar{x}, \quad q=-\frac{1}{\sqrt{\gamma}},
$$

we brought into the form of the canonical equation

$$
\frac{d^{4} u}{d t^{4}}+q \frac{d^{2} u}{d t^{2}}+u^{3}-u=0 .
$$

Denoting $x=u, y=\frac{d u}{d t}, z=\frac{d^{2} u}{d t^{2}}, v=\frac{d^{3} u}{d t^{3}}$ we get the polynomial differential system

$$
\dot{x}=y, \quad \dot{y}=z, \quad \dot{z}=v, \quad \dot{v}=x-q z-x^{3},
$$

with $(x, y, z, v) \in \mathbb{R}^{4}$ and $q \in \mathbb{R}$ negative.

Besides the large amount of papers concerning the Fisher-Kolmogorov and Extended Fisher-Kolmogorov equations existing on the literature (see for instance $[1,7,8])$, there are a few works describing their dynamics. The aim of this note is to describe the global dynamics of stationary solutions of the EFK-equation, more precisely to characterize all the $\alpha$ - and $\omega$-limit sets of all orbits of this equation. Before doing it let us remember some basic results about symmetric and reversible systems, which shall be used later on in the study of system (2).

Let

$$
\dot{x}=F(x), \quad x \in \mathbb{R}^{n},
$$

be a smooth differential system and $S: \mathbb{R}^{n} \rightarrow \mathbb{R}^{n}, S(x)=y$ be a linear map satisfying $S \circ S=I d$. We say that (3) is symmetric with respect to $S$ if $\dot{y}(t)=F(y(t))$. We say that (3) is reversible with respect to $S$ if $\dot{y}(t)=-F(y(t))$.

We point out some properties of symmetric and reversible systems.

(a) Their phase portraits are symmetric with respect to

$$
\operatorname{Fix}(S)=\left\{x \in \mathbb{R}^{n}: S(x)=x\right\} .
$$

(b) If $x(t)$ is a solution of system (3), then $S(x(t))=y(t)$ is a solution of (3) for the symmetric case, and $S(x(t))=y(-t)$ is a solution of (3) for the reversible case.

For the reversible case:

(c1) Any orbit meeting $\operatorname{Fix}(S)$ at two different points is a periodic orbit.

(c2) Any equilibrium point or periodic orbit on $\operatorname{Fix}(S)$ cannot be an attractor or a repeller. 
(c3) Intersection of (un)-stable manifolds with $\operatorname{Fix}(S)$ imply the existence of heteroclinic or homoclinic orbits.

Our first result about system (2) is the following.

Theorem 1. The following statements hold for system (2).

(a) It is reversible with respect to the involution

$$
R(x, y, z, v)=(x,-y, z,-v) .
$$

(b) It has the first integral

$$
H(x, y, z, v)=\frac{q}{2} y^{2}-\frac{x^{2}}{2}-\frac{z^{2}}{2}+v y+\frac{x^{4}}{4} .
$$

For $h \neq 0$ and $h \neq-1 / 4$ the set $H^{-1}(h)$ is a smooth 3-dimensional manifold.

(c) The flow of system (2) on $H^{-1}(h) \cap\left(\mathbb{R}^{4} \backslash\{y=0\}\right)$ is determined by the constrained 3-dimensional differential system

$$
\dot{x}=y, \quad \dot{y}=z, \quad 4 y \dot{z}=4 h+2 x^{2}-x^{4}-2 q y^{2}+2 z^{2} .
$$

(d) The equilibrium points of system (2) are $(0,0,0,0) \in H^{-1}(0)$ and $( \pm 1,0,0,0) \in H^{-1}(-1 / 4)$.

The plane $y=0$ is an impasse surface for system (4) according to the terminology used in [6,9]. Under the rescaling $d t=4 y d \tau$ we transform system (4) into the regularized vector field given by

$$
\dot{x}=4 y^{2}, \quad \dot{y}=4 y z, \quad \dot{z}=4 h+2 x^{2}-x^{4}-2 q y^{2}+2 z^{2} .
$$

As any polynomial differential system, equations (5) can be extended to an analytic system on a closed ball of radius one, whose interior is diffeomorphic to $\mathbb{R}^{3}$ and its boundary, the 2-dimensional sphere $\mathbb{S}^{2}$, plays the role of the infinity. This closed ball is denoted by $\mathbb{D}^{3}$ and called the Poincaré ball, because the technique for doing such an extension is precisely the Poincaré compactification for a polynomial differential system in $\mathbb{R}^{3}$, which is described in details in [2] and a summary of it is given in section 3 ahead. By using this compactification technique the dynamics of system (5) at infinity is studied and we have the following result.

Theorem 2. For all values of the parameters $h, q \in \mathbb{R}$ the phase portrait of system (5) on the sphere of infinity is as shown in figure 1.

We say that a set $V \subseteq \mathbb{D}^{3}$ is invariant by the flow of (5) if for any $p \in V$ the whole orbit passing through $p$ is contained in $V$. The sphere of the infinity is always an invariant set.

Let $\varphi(t)=\varphi(t, p)$ be the solution of the compactified system (5) passing through the point $p \in \mathbb{D}^{3}$, defined on its maximal interval $I_{p}=\mathbb{R}$, because $\mathbb{D}^{3}$ is compact. Then the $\alpha$-limit set of $p$ is the invariant set

$$
\alpha(p)=\left\{q \in \mathbb{D}^{3}: \exists\left\{t_{n}\right\} \text { such that } t_{n} \rightarrow-\infty \text { and } \varphi\left(t_{n}\right) \rightarrow q \text { as } n \rightarrow \infty\right\} .
$$




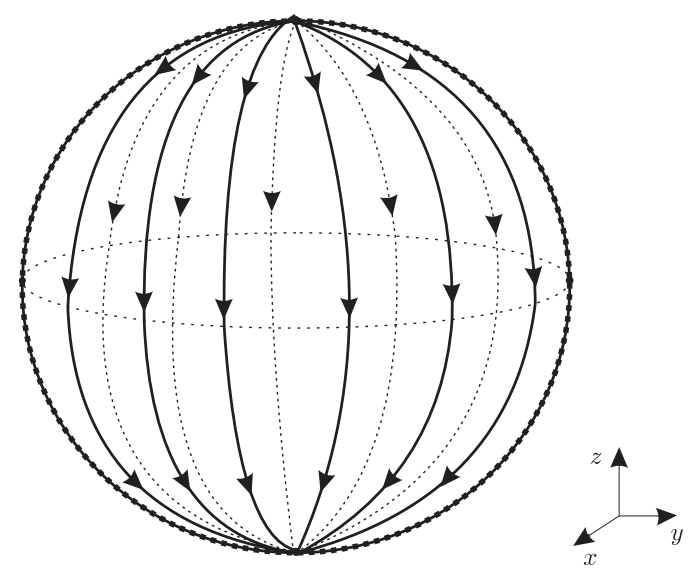

Figure 1. Phase portrait of system (5) at the infinity of the Poincar ball: there is a circle of equilibrium points containing the endpoints of the $y z$-plane; the positive (negative) endpoints of the $z$-axis behave like unstable (stable) nonhyperbolic improper nodes.

In a similar way, the $\omega$-limit set of $p$ is the invariant set

$\omega(p)=\left\{q \in \mathbb{D}^{3}: \exists\left\{t_{n}\right\}\right.$ such that $t_{n} \rightarrow \infty$ and $\varphi\left(t_{n}\right) \rightarrow q$ as $\left.n \rightarrow \infty\right\}$.

We also study the phase portrait of system (5) on the Poincar ball. In order to state our next results, we introduce the notation:

- $\mathbb{D}_{++}^{3}=\left\{(x, y, z) \in \mathbb{R}^{3}: x^{2}+y^{2}+z^{2}<1, y>0, z>0\right\}$ and $\mathbb{D}_{+-}^{3}=\left\{(x, y, z) \in \mathbb{R}^{3}: x^{2}+y^{2}+z^{2}<1, y>0, z<0\right\}$

- $\mathcal{A}=\left\{(x, y, z) \in \mathbb{D}^{3}: x^{2}+y^{2}+z^{2}<1, y>0\right\}$

- $L_{N}=\left\{x=0,0 \leq y \leq 1, y^{2}+z^{2}=1\right\}$ and $L_{S}=\{x=0,-1 \leq y \leq$ $\left.0, y^{2}+z^{2}=1\right\}$.

We denote by $\mathcal{S}_{h q}$ the closure of the surface $\dot{z}=4 h+2 x^{2}-x^{4}-2 q y^{2}+2 z^{2}=$ 0 in the Poincar ball. We also denote by $\mathcal{S}_{h}$ the closure of $\mathcal{S}_{h q} \cap\{y=0\}$ in the Poincar ball.

We remember that if $C \subseteq \mathbb{D}^{3}$ then $\partial C$ denotes its boundary and $\bar{C}$ denotes its closure in the Poincar ball.

Theorem 3. The polynomial differential system (5) in the Poincar ball satisfies the following statements.

(a) It is symmetric with respect to the involution $S(x, y, z)=(x,-y, z)$, and reversible with respect to the involution $R(x, y, z)=(-x,-y,-z)$.

(b) The plane $y=0$ is invariant by the flow. The set of all finite equilibrium points of system (5) is the finite part of the curve $\mathcal{S}_{h}$. The phase portrait on $y=0$ is as it is shown in figure 2.

(c) The eigenvalues of the linear part of system (5) at the point $(x, 0, z)$ are $0,4 z$ and $4 z$.

(d) System (5) has no periodic orbits. 
(e) If $p \in \mathcal{A}$ then $\alpha(p)$ and $\omega(p)$ are contained in the set of equilibrium points contained in $\partial \mathcal{A}$.

(f) If $p \in \mathcal{A}$ then

$$
\begin{aligned}
& -\alpha(p) \cap\left(L_{N} \backslash\{(0,0,1),(0,1,0)\}\right)=\emptyset, \\
& -\alpha(p) \cap\left(\mathcal{S}_{h} \cap\{-1<z<0\}\right)=\emptyset, \\
& -\omega(p) \cap\left(L_{S} \backslash\{(0,0,-1),(0,1,0)\}\right)=\emptyset \text { and } \\
& -\omega(p) \cap\left(\mathcal{S}_{h} \cap\{0<z<1\}\right)=\emptyset .
\end{aligned}
$$

(g) If $\alpha(p)$ (resp. $\omega(p))$ is contained in $\left(\mathcal{S}_{h} \cap\{-1<z<1, z \neq 0\}\right)$, then $\alpha(p)$ (resp. $\omega(p))$ is formed by only one equilibrium point.

Remark. According to statement (a) of Theorem 3 it is enough to describe the phase portrait of system (5) only on $\overline{\mathbb{D}_{++}^{3}}$. But since $\overline{\mathbb{D}_{++}^{3}}$ is not invariant by the flow of (5) and the minimal compact invariant set containing $\overline{\mathbb{D}_{++}^{3}}$ is $\overline{\mathcal{A}}$, we shall describe all the $\alpha-$ and $\omega$-limit sets of the orbits contained in $\overline{\mathcal{A}}$.

Theorem 4. The $\alpha$-and $\omega$-limit sets of the solutions of the compactified system (5) satisfy the following statements.

(a) If $p \in \partial \mathcal{A}$ then $\alpha(p)$ and $\omega(p)$ are completely characterized in figures 1 and 2.

(b) The surface $\mathcal{S}_{h q}$ is the boundary separating two open regions $\mathcal{Z}^{+}=$ $\{(x, y, z) \in \mathcal{A}: \dot{z}>0\}$ and $\mathcal{Z}^{-}=\{(x, y, z) \in \mathcal{A}: \dot{z}<0\}$. If $p \in \mathcal{Z}^{+}$is such that the whole orbit passing through $p$ is contained in $\mathcal{Z}^{+}$, then $\omega(p) \subset L_{N}$. If $p \in \mathcal{Z}^{-}$is such that the whole orbit passing through $p$ is contained in $\mathcal{Z}^{-}$, then $\alpha(p) \subset \mathcal{S}_{h} \cap\{z \geq 0\}$ and $\omega(p) \subset \mathcal{S}_{h} \cap\{z \leq 0\}$.

(c) The boundary of the surface $\mathcal{S}_{h q}$ at infinity is the great circle $x=0$.

If the orbit through $p \in \mathcal{A}$ intersects $\mathcal{S}_{h q}$ a more detailed analysis is necessary.

Remark. We emphasize that the conclusions of Theorems 2, 3 and 4 are concerned to the dynamics of the differential system (5) and its compactification. The orbits of the original differential system, i.e. system (2), are contained in the hyper surfaces $H^{-1}(h)$ where $H=\frac{q}{2} y^{2}-\frac{x^{2}}{2}-\frac{z^{2}}{2}+v y+\frac{x^{4}}{4}$. System (2) on $H^{-1}(h) \cap\{y \neq 0\}$ is topologically equivalent to system (5) on $\{y \neq 0\}$ that is, removing from its orbits the impasse points, and inverting the orientation of the orbits contained on $\{y<0\}$. No additional information is given for the orbits of system (2) passing through $\{y=0\}$. Due to this fact, in spite of system (5) has no periodic orbits, our analysis is not sufficient to detect periodic orbits of the original system if they cross $\{y=0\}$. A way to study the complete phase portrait is to solve $H=0$ on the variable $z$ and study the corresponding two differential systems according the sign of the square root which appears after the substitution of the 

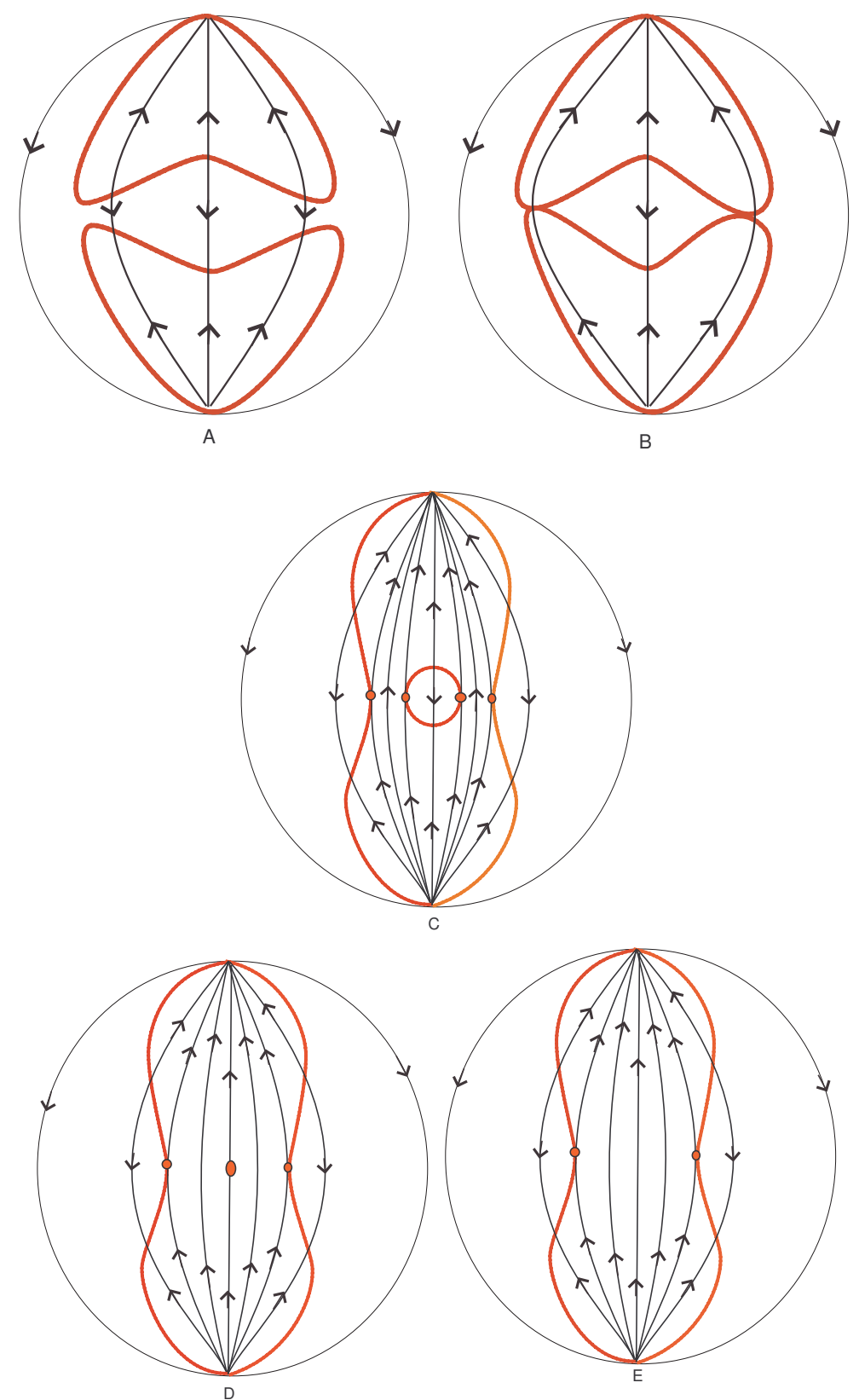

Figure 2. Global phase portrait of system (5) on the disk $\{y=$ $0\} \cap \mathbb{D}^{3}:$ (A) $h<-\frac{1}{4}$, (B) $h=-\frac{1}{4}$, (C) $-\frac{1}{4}<h<0$, (D) $h=0$, (E) $h>0$. The bold line is formed by equilibrium points.

variable $z$. But to do this work is equivalent to write another paper longer than this one. 
The paper is organized as follows. In section 2 we prove Theorem 1 and study the linear part of the differential system (2) at the equilibrium points. In section 3 we give a summary of the formulas related with the Poincaré compactification of a polynomial vector field in $\mathbb{R}^{3}$, because they will be used along this paper. We also study how the invariant algebraic surfaces $H^{-1}(h)$ extend to infinity in the Poincaré ball (see Lemma 6). In section 4 we prove Theorem 2, and in section 5 we prove Theorem 3 . In section 6 we prove Theorem 4 and we study the $\alpha$ - and $\omega$-limit sets when the parameter $h$ varies.

\section{Proof of Theorem 1}

(a) Denote $X(x, y, z, v)=\left(y, z, v, x-q z-x^{3}\right)$. If

$$
\left(y_{1}, y_{2}, y_{3}, y_{4}\right)=R(x, y, z, v) \quad \text { and } \quad(\dot{x}, \dot{y}, \dot{z}, \dot{v})=X(x, y, z, v)
$$

then $\left(\dot{y}_{1}, \dot{y_{2}}, \dot{y_{3}}, \dot{y}_{4}\right)=-X\left(y_{1}, y_{2}, y_{3}, y_{4}\right)$. Thus (a) is proved.

(b) Since

$$
\frac{d H}{d t}=H_{x} \dot{x}+H_{y} \dot{y}+H_{z} \dot{z}+H_{v} \dot{v}=0,
$$

it follows that $H$ is a first integral of system (2). The gradient of $H$ is given by

$$
\nabla H(x, y, z, v)=\left(-x+x^{3}, q y+v,-z, y\right),
$$

which is equal to $(0,0,0,0)$ if and only if $x=0,1,-1$ and $y=z=v=0$. Since $H(0,0,0,0)=0$ and $H( \pm 1,0,0,0)=-\frac{1}{4}$ each level $H^{-1}(h), h \neq 0,-\frac{1}{4}$ is a 3 -dimensional invariant manifold of system (2) on $\mathbb{R}^{4}$. This proves statements (b) and (d).

(c) The dynamics on each level $H^{-1}(h)$ excepted on the surface $H^{-1}(h) \cap$ $\{y=0\}$ is determined by the constrained system (4). In fact, on $H^{-1}(h) \cap$ $\left(\mathbb{R}^{4} \backslash\{y=0\}\right)$

$$
v=\frac{4 h+2 x^{2}-x^{4}-2 q y^{2}+2 z^{2}}{4 y} .
$$

Thus system (2) becomes system (4).

The orbits of system (4) are defined only outside the impasse hypersurface by the corresponding similar elements of system (5). Note that the phase portrait of system (4) is the same as of system (5) by removing from its orbits the impasse points and inverting the orientation of the orbits contained in $y<0$.

Now we study the linear part of the differential system (2) at the equilibrium points.

Proposition 5. Consider system (2). 
(a) For any $q \in \mathbb{R}$, there exist $\lambda>0, \mu>0$ such that the eigenvalues at $(0,0,0,0)$ are $\pm \lambda \in \mathbb{R}$ and $\pm \mu i$.

(b) The eigenvalues at $( \pm 1,0,0,0)$ are

(b1) $\pm \lambda \in \mathbb{R}$ and $\pm \nu \in \mathbb{R}$, with $\lambda \nu \neq 0$, if $q \in(-\infty,-\sqrt{8})$;

(b2) $\pm \lambda \in \mathbb{R} \backslash\{0\}$ with algebraic multiplicities equal to 2 , if $q=-\sqrt{8}$;

(b3) $\pm a \pm b i$, with $a b \neq 0$, if $q \in(-\sqrt{8}, \sqrt{8})$;

(b4) $\pm \mu i$ with algebraic multiplicities equal to 2 and $\mu \neq 0$, if $q=\sqrt{8}$;

(b5) $\pm \mu i$ and $\pm \nu i$ with $\mu \nu \neq 0$, if $q \in(\sqrt{8}, \infty)$.

Proof. Denote by $X(x, y, z, v)=\left(y, z, v, x-q z-x^{3}\right)$. We have

$$
D X(0,0,0,0)=\left(\begin{array}{cccc}
0 & 1 & 0 & 0 \\
0 & 0 & 1 & 0 \\
0 & 0 & 0 & 1 \\
1 & 0 & -q & 0
\end{array}\right) .
$$

The eigenvalues of $D X(0,0,0,0)$ are

$$
\pm \frac{\sqrt{-2 q+2 \sqrt{q^{2}+4}}}{2}, \pm \frac{\sqrt{-2 q-2 \sqrt{q^{2}+4}}}{2} .
$$

Since for any $q \in \mathbb{R}$ we have that $-2 q+2 \sqrt{q^{2}+4}>0$ and $-2 q-2 \sqrt{q^{2}+4}<$ 0 statement (a) is verified. We also have

$$
D X( \pm 1,0,0,0)=\left(\begin{array}{cccc}
0 & 1 & 0 & 0 \\
0 & 0 & 1 & 0 \\
0 & 0 & 0 & 1 \\
-2 & 0 & -q & 0
\end{array}\right) .
$$

The eigenvalues of $D X( \pm 1,0,0,0)$ are

$$
\pm \frac{\sqrt{-2 q+2 \sqrt{q^{2}-8}}}{2}, \pm \frac{\sqrt{-2 q-2 \sqrt{q^{2}-8}}}{2} .
$$

If $q \in(-\infty,-\sqrt{8})$ then both $-2 q \pm 2 \sqrt{q^{2}-8}$ are positive. If $q=-\sqrt{8}$ then $-2 q \pm 2 \sqrt{q^{2}-8}=-2 q>0$. If $q \in(-\sqrt{8}, \sqrt{8})$ then both $-2 q \pm 2 \sqrt{q^{2}-8}$ are non real. If $q=\sqrt{8}$ then $-2 q \pm 2 \sqrt{q^{2}-8}=-2 q<0$. If $q \in(\sqrt{8}, \infty)$ then both $-2 q \pm 2 \sqrt{q^{2}-8}$ are negative.

\section{The Poincaré Compactification in $\mathbb{R}^{3}$}

A polynomial vector field $X$ in $\mathbb{R}^{n}$ can be extended to a unique analytic vector field on the sphere $\mathbb{S}^{n}$. The technique for making such an extension is called the Poincaré compactification and allows us to study a polynomial vector field in a neighborhood of infinity, which corresponds to the equator $\mathbb{S}^{n-1}$ of the sphere $\mathbb{S}^{n}$. Poincare introduced this compactification for polynomial vector fields in $\mathbb{R}^{2}$. Its extension to $\mathbb{R}^{n}$ for $n>2$ can be found in [2] and some applications in $[4,5]$. In this section we describe the Poincaré 
compactification for polynomial vector fields in $\mathbb{R}^{3}$ following closely what is made in [2].

In $\mathbb{R}^{3}$ we consider the polynomial differential system

$$
\dot{x}=P^{1}(x, y, z), \quad \dot{y}=P^{2}(x, y, z), \quad \dot{z}=P^{3}(x, y, z),
$$

or equivalently its associated polynomial vector field $X=\left(P^{1}, P^{2}, P^{3}\right)$. The degree $n$ of $X$ is defined as $n=\max \left\{\operatorname{deg}\left(P^{i}\right): i=1,2,3\right\}$.

Let $\mathbb{S}^{3}=\left\{y=\left(y_{1}, y_{2}, y_{3}, y_{4}\right) \in \mathbb{R}^{4}:\|y\|=1\right\}$ be the unit sphere in $\mathbb{R}^{4}$, and $\mathbb{S}_{+}=\left\{y \in \mathbb{S}^{3}: y_{4}>0\right\}$ and $\mathbb{S}_{-}=\left\{y \in \mathbb{S}^{3}: y_{4}<0\right\}$ be the northern and southern hemispheres of $\mathbb{S}^{4}$ respectively. The tangent space to $\mathbb{S}^{3}$ at the point $y$ is denoted by $T_{y} \mathbb{S}^{3}$. Then the tangent plane

$$
T_{(0,0,0,1)} \mathbb{S}^{3}=\left\{\left(x_{1}, x_{2}, x_{3}, 1\right) \in \mathbb{R}^{4}:\left(x_{1}, x_{2}, x_{3}\right) \in \mathbb{R}^{3}\right\}
$$

is identified with $\mathbb{R}^{3}$.

We consider the central projections $f_{+}: \mathbb{R}^{3}=T_{(0,0,0,1)} \mathbb{S}^{3} \longrightarrow \mathbb{S}_{+}$and $f_{-}: \mathbb{R}^{3}=T_{(0,0,0,1)} \mathbb{S}^{3} \longrightarrow \mathbb{S}_{-}$defined by $f_{ \pm}(x)= \pm\left(x_{1}, x_{2}, x_{3}, 1\right) / \Delta x$, where $\Delta x=\left(1+\sum_{i=1}^{3} x_{i}^{2}\right)^{1 / 2}$. Through these central projections $\mathbb{R}^{3}$ is identified with the northern and southern hemispheres. The equator of $\mathbb{S}^{3}$ is $\mathbb{S}^{2}=$ $\left\{y \in \mathbb{S}^{3}: y_{4}=0\right\}$. Clearly $\mathbb{S}^{2}$ can be identified with the infinity of $\mathbb{R}^{3}$.

The maps $f_{+}$and $f_{-}$define two copies of $X$ on $\mathbb{S}^{3}$, one $D f_{+} \circ X$ in the northern hemisphere and the other $D f_{-} \circ X$ in the southern one. Denote by $\bar{X}$ the vector field on $\mathbb{S}^{3} \backslash \mathbb{S}^{2}=\mathbb{S}_{+} \cup \mathbb{S}_{-}$which, restricted to $\mathbb{S}_{+}$coincides with $D f_{+} \circ X$ and restricted to $\mathbb{S}_{-}$coincides with $D f_{-} \circ X$.

The expression for $\bar{X}(y)$ on $\mathbb{S}_{+} \cup \mathbb{S}_{-}$is

$$
\bar{X}(y)=y_{4}\left(\begin{array}{lll}
1-y_{1}^{2} & -y_{2} y_{1} & -y_{3} y_{1} \\
-y_{1} y_{2} & 1-y_{2}^{2} & -y_{3} y_{2} \\
-y_{1} y_{3} & -y_{2} y_{3} & 1-y_{3}^{2} \\
-y_{1} y_{4} & -y_{2} y_{4} & -y_{3} y_{4}
\end{array}\right)\left(\begin{array}{l}
P^{1} \\
P^{2} \\
P^{3}
\end{array}\right),
$$

where $P^{i}=P^{i}\left(y_{1} /\left|y_{4}\right|, y_{2} /\left|y_{4}\right|, y_{3} /\left|y_{4}\right|\right)$. Written in this way $\bar{X}(y)$ is a vector field in $\mathbb{R}^{4}$ tangent to the sphere $\mathbb{S}^{3}$.

Now we can extend analytically the vector field $\bar{X}(y)$ to the whole sphere $\mathbb{S}^{3}$ by $p(X)(y)=y_{4}^{n-1} \bar{X}(y)$. This extended vector field $p(X)$ is called the Poincaré compactification of $X$ on $\mathbb{S}^{3}$.

As $\mathbb{S}^{3}$ is a differentiable manifold in order to compute the expression for $p(X)$ we can consider the eight local charts $\left(U_{i}, F_{i}\right),\left(V_{i}, G_{i}\right)$, where $U_{i}=$ $\left\{y \in \mathbb{S}^{3}: y_{i}>0\right\}$ and $V_{i}=\left\{y \in \mathbb{S}^{3}: y_{i}<0\right\}$ for $i=1,2,3,4$; the diffeomorphisms $F_{i}: U_{i} \rightarrow \mathbb{R}^{3}$ and $G_{i}: V_{i} \rightarrow \mathbb{R}^{3}$ for $i=1,2,3,4$ are the inverses of the central projections from the origin to the tangent planes at the points $( \pm 1,0,0,0),(0, \pm 1,0,0),(0,0, \pm 1,0)$ and $(0,0,0, \pm 1)$, respectively. Now we do the computations on $U_{1}$. Suppose that the origin $(0,0,0,0)$, the point $\left(y_{1}, y_{2}, y_{3}, y_{4}\right) \in \mathbb{S}^{3}$ and the point $\left(1, z_{1}, z_{2}, z_{3}\right)$ in the tangent plane to $\mathbb{S}^{3}$ at $(1,0,0,0)$ are collinear. Then we have $1 / y_{1}=z_{1} / y_{2}=z_{2} / y_{3}=$ 
$z_{3} / y_{4}$, and consequently $F_{1}(y)=\left(y_{2} / y_{1}, y_{3} / y_{1}, y_{4} / y_{1}\right)=\left(z_{1}, z_{2}, z_{3}\right)$ defines the coordinates on $U_{1}$. As

$$
D F_{1}(y)=\left(\begin{array}{cccc}
-y_{2} / y_{1}^{2} & 1 / y_{1} & 0 & 0 \\
-y_{3} / y_{1}^{2} & 0 & 1 / y_{1} & 0 \\
-y_{4} / y_{1}^{2} & 0 & 0 & 1 / y_{1}
\end{array}\right)
$$

and $y_{4}^{n-1}=\left(z_{3} / \Delta z\right)^{n-1}$, the analytical vector field $p(X)$ becomes

$$
\frac{z_{3}^{n}}{(\Delta z)^{n-1}}\left(-z_{1} P^{1}+P^{2},-z_{2} P^{1}+P^{3},-z_{3} P^{1}\right),
$$

where $P^{i}=P^{i}\left(1 / z_{3}, z_{1} / z_{3}, z_{2} / z_{3}\right)$.

In a similar way we can deduce the expressions of $p(X)$ in $U_{2}$ and $U_{3}$. These are

$$
\frac{z_{3}^{n}}{(\Delta z)^{n-1}}\left(-z_{1} P^{2}+P^{1},-z_{2} P^{2}+P^{3},-z_{3} P^{2}\right),
$$

where $P^{i}=P^{i}\left(z_{1} / z_{3}, 1 / z_{3}, z_{2} / z_{3}\right)$ in $U_{2}$, and

$$
\frac{z_{3}^{n}}{(\Delta z)^{n-1}}\left(-z_{1} P^{3}+P^{1},-z_{2} P^{3}+P^{2},-z_{3} P^{3}\right),
$$

where $P^{i}=P^{i}\left(z_{1} / z_{3}, z_{2} / z_{3}, 1 / z_{3}\right)$ in $U_{3}$.

The expression for $p(X)$ in $U_{4}$ is $z_{3}^{n+1}\left(P^{1}, P^{2}, P^{3}\right)$, now denoting $P^{i}=$ $P^{i}\left(z_{1}, z_{2}, z_{3}\right)$. The expression for $p(X)$ in the local chart $V_{i}$ is the same as in $U_{i}$ multiplied by $(-1)^{n-1}$.

When we work with the expression of the compactified vector field $p(X)$ in the local charts we usually omit the factor $1 /(\Delta z)^{n-1}$. We can do that through a rescaling of the time variable.

In what follows we shall work with the orthogonal projection of $p(X)$ from the closed northern hemisphere to $y_{4}=0$, we continue denoting this projected vector field by $p(X)$. Note that the projection of the closed northern hemisphere is a closed ball $B$ of radius one, whose interior is diffeomorphic to $\mathbb{R}^{3}$ and whose boundary $\mathbb{S}^{2}$ corresponds to the infinity of $\mathbb{R}^{3}$. Of course $p(X)$ is defined in the whole closed ball $\mathbb{D}^{3}$ in such a way that the flow on the boundary is invariant. This new vector field on $\mathbb{D}^{3}$ will be called the Poincaré compactification of $X$, and $\mathbb{D}^{3}$ will be called the Poincaré ball.

Remark. All the points on the invariant sphere $\mathbb{S}^{2}$ at infinity in the coordinates of any local chart $U_{i}$ and $V_{i}$ have $z_{3}=0$. Also, the points in the interior of the Poincare ball, which is diffeomorphic to $\mathbb{R}^{3}$, are given in the local charts $U_{1}, U_{2}$ and $U_{3}$ by $z_{3}>0$ and in the local charts $V_{1}, V_{2}$ and $V_{3}$ by $z_{3}<0$.

Lemma 6. Let $f\left(x_{1}, x_{2}, x_{3}\right)=0$ be an algebraic surface of $\mathbb{R}^{3}=T_{(0,0,0,1)} \mathbb{S}^{3}$ of degree $m$. The extension of this surface to the boundary of the Poincaré ball is obtained solving the system

$$
y_{4}^{m} f\left(\frac{x_{1}}{y_{4}}, \frac{x_{2}}{y_{4}}, \frac{x_{3}}{y_{4}}\right)=0, \quad y_{4}=0 .
$$


Proof. We project the northern hemisphere $y_{4}>0$ of the sphere $\mathbb{S}^{3}$ on $y_{4}=0$, i.e. on the Poincaré ball using the equations

$$
x_{1}=\frac{y_{1}}{y_{4}}, \quad x_{2}=\frac{y_{2}}{y_{4}}, \quad x_{3}=\frac{y_{3}}{y_{4}} .
$$

Thus the points on the infinity correspond to the points on the equator $y_{4}=0$ of $\mathbb{S}^{3}$.

\section{Proof of Theorem 2}

In this section we shall make an analysis of the flow of system (5) near and at infinity. In order to do it in the next three subsections we shall analyze the Poincaré compactification of system (5) in the local charts $U_{i}$ and $V_{i}$, $i=1,2,3$ as described in section 3 and in section 4.4 we put together the results obtained to obtain the proof of Theorem 2 .

4.1. In the local charts $\mathrm{U}_{1}$ and $\mathrm{V}_{1}$. From the results of section 3 the expression of the Poincaré compactification $p(X)$ of system (5) in the local chart $U_{1}$ is given by

$$
\begin{aligned}
& \dot{z}_{1}=-4 z_{1} z_{3}^{2}\left(z_{1}^{2}-z_{2}\right), \\
& \dot{z}_{2}=-1-4 z_{1}^{2} z_{2} z_{3}^{2}+4 h z_{3}^{4}+2 z_{3}^{2}-2 q z_{1}^{2} z_{3}^{2}+2 z_{2}^{2} z_{3}^{2}, \\
& \dot{z}_{3}=-4 z_{1}^{2} z_{3}^{2} .
\end{aligned}
$$

For $z_{3}=0$ (which corresponds to the points on the sphere $\mathbb{S}^{2}$ of the infinity) (6) reduces to

$$
\dot{z}_{1}=0 \quad \dot{z}_{2}=-1
$$

from which follows that system (5) has no equilibrium point nor periodic orbits in the portion of the Poincaré sphere parametrized by the local chart $U_{1}$, which contains the positive endpoint of the $x$-axis. It implies that there are no trajectories of system (5) which tend to or come from infinity trough this part of the sphere, where the dynamics is given by system (7), which is trivial.

The flow in the local chart $V_{1}$ is the same as the flow in the local chart $U_{1}$ reversing appropriately the time, since the compactified vector field $p(X)$ in $V_{1}$ coincides with the vector field $p(X)$ in $U_{1}$ multiplied by $(-1)^{n-1}$, where $n=4$ is the degree of system (5) (for details see section 3). Hence system (5) also has a trivial dynamics on the portion of the infinite sphere parametrized by the local chart $U_{2}$, which contains negative endpoint of the $x$-axis. Actually, this dynamics is given by the system

$$
\dot{z}_{1}=0 \quad \dot{z}_{2}=1 \text {. }
$$

See figure 1 which shows the dynamics of system (5) on the Poincaré sphere for a view of the dynamics on the portions of this sphere, containing the endpoints of the $x$-axis, described above. 
4.2. In the local charts $\mathrm{U}_{2}$ and $\mathrm{V}_{2}$. Again using the results of section 3 we have the expression of the Poincaré compactification $p(X)$ of system (5) in the local chart $U_{2}$, which is given by

$$
\begin{aligned}
& \dot{z}_{1}=-4 z_{3}^{2}\left(z_{1} z_{2}-1\right), \\
& \dot{z}_{2}=-2 z_{2}^{2} z_{3}^{2}+4 h z_{3}^{4}+2 z_{1}^{2} z_{3}^{2}-z_{1}^{4}-2 q z_{3}^{2}, \\
& \dot{z}_{3}=-4 z_{2} z_{3}^{2} .
\end{aligned}
$$

For $z_{3}=0$ (which corresponds to the points on the sphere $\mathbb{S}^{2}$ of the infinity) system (8) has a line of equilibria given by the $z_{2}$-axis and the linear part of the system at these equilibria has three null eigenvalues. Let us study the flow near these line of equilibria. From the compactification procedure described in section 3 follows that the $z_{1} z_{2}$-plane is invariant under the flow of (8), so we can completely describe the dynamics on the sphere at infinity. In fact, if $z_{3}=0$ system (8) restricted to the $z_{1} z_{2}$-plane is given by

$$
\dot{z}_{1}=0, \quad \dot{z}_{2}=-z_{1}^{4} .
$$

Hence the phase portrait of system (8) restricted to this plane is as shown in figure 3 (a). See also figure 1 which shows the global phase portrait of system (5) on the Poincar sphere.
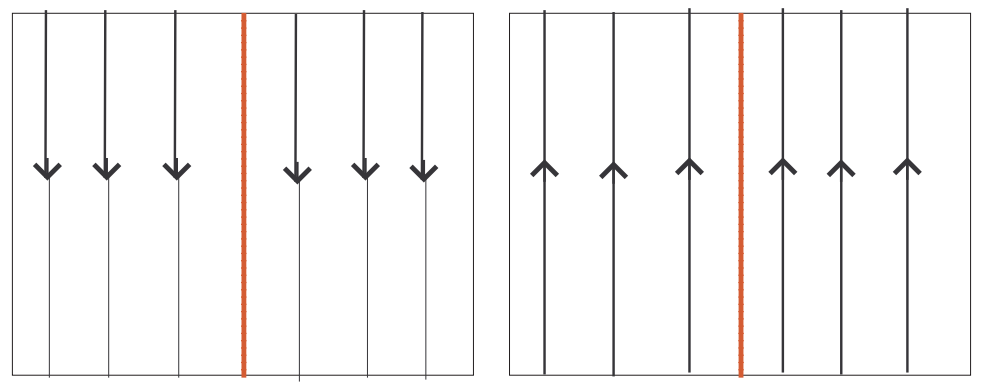

(a) $U_{2}$

(b) $V_{2}$

Figure 3. Dynamics of system (5) on the sphere of the infinity in the local charts $U_{2}$ (a) and $V_{2}$ (b). There is a line of equilibria in the $z_{2}$-axis.

The flow in the local chart $V_{2}$ is the same as the flow in the local chart $U_{2}$ reversing the time (see figure $3(\mathrm{~b})$ ), because the compactified vector field $p(X)$ in $V_{2}$ coincides with the vector field $p(X)$ in $U_{2}$ multiplied by $(-1)^{n-1}$, where $n=4$ is the degree o system (5). 
4.3. In the local charts $\mathrm{U}_{3}$ and $\mathrm{V}_{3}$. The expression of the Poincar compactification $p(X)$ of system (5) in the local chart $U_{3}$ is

$$
\begin{aligned}
& \dot{z}_{1}=-4 h z_{1} z_{3}^{4}-2 z_{1}^{3} z_{3}^{2}+z_{1}^{5}+2 q z_{1} z_{2}^{2} z_{3}^{2}-2 z_{1} z_{3}^{2}+4 z_{2}^{2} z_{3}^{2} \\
& \dot{z}_{2}=-4 h z_{2} z_{3}^{4}-2 z_{1}^{2} z_{2} z_{3}^{2}+z_{1}^{4} z_{2}+2 q z_{2}^{3} z_{3}^{2}+2 z_{2} z_{3}^{2}, \\
& \dot{z}_{3}=-4 h z_{3}^{5}-2 z_{1}^{2} z_{3}^{3}+z_{1}^{4} z_{3}+2 q z_{2}^{2} z_{3}^{3}-2 z_{3}^{3} .
\end{aligned}
$$

For $z_{3}=0$, system (10) restricted to the invariant $z_{1} z_{2}$-plane reduces to

$$
\dot{z}_{1}=z_{1}^{5}, \quad \dot{z_{2}}=z_{2} z_{1}^{4} .
$$

The solutions of this system behave like shown in figure 4 (a), which corresponds to the dynamics of system (5) at infinity in the local chart $U_{3}$. The dynamics at infinity in the chart $V_{3}$ is as shown in shown in figure 4 (b). Indeed for $z_{1} \neq 0$ the system is equivalent to

$$
\dot{z}_{1}=z_{1}, \quad \dot{z}_{2}=z_{2}
$$

whose origin is an improper node. The set $\left\{z_{1}=0\right\}$ determines a line of equilibria. See also figure 1.

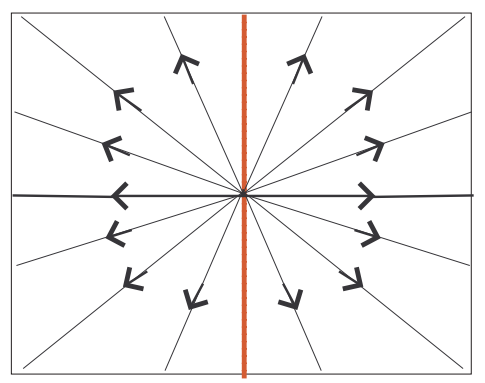

(a) $U_{3}$

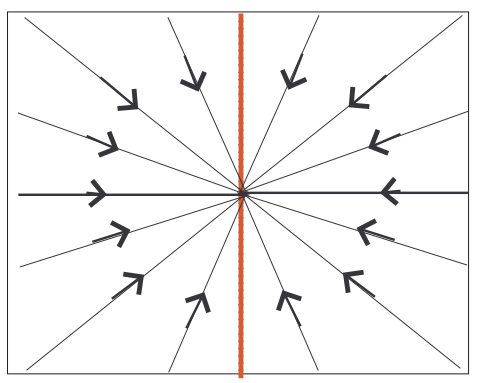

(b) $V_{3}$

FiguRE 4. Dynamics of system (5) on the sphere of the infinity in the local charts $U_{3}$ (a) and $V_{3}$ (b). There is a line of equilibria in the $z_{2}$-axis.

4.4. Dynamics of system (5) on the Poincar sphere of the infinity. Considering the analysis made in the previous subsections we have a global picture of the dynamical behavior of system (5) on the sphere at infinity. The system has a line of (nonhyperbolic) equilibria containing the endpoints of the $y z$-plane and there are no more equilibrium points on the sphere. The equilibria at the endpoints of the $z$-axis behave like improper nodes, even being nonhyperbolic. The global dynamics on the sphere of the infinity, 
constructed based on the calculations in the local charts $U_{i}$ and $V_{i}, i=1,2,3$, is shown in figure 1.

We observe that the description of the complete phase portrait of system (5) on the sphere at infinity was possible because of the invariance of this set under the flow of the compactified system, since the dynamics near the line of equilibria is highly degenerate.

\section{Proof of Theorem 3}

We denote $Y(x, y, z)=\left(4 y^{2}, 4 y z, 4 h+2 x^{2}-x^{4}-2 q y^{2}+2 z^{2}\right)$.

Proof of Theorem 3. (a) If $\left(y_{1}, y_{2}, y_{3}\right)=S(x, y, z)$ and $(\dot{x}, \dot{y}, \dot{z})=Y(x, y, z)$ then $\left(\dot{y}_{1}, \dot{y_{2}}, \dot{y_{3}}\right)=Y\left(y_{1}, y_{2}, y_{3}\right)$. If $\left(y_{1}, y_{2}, y_{3}\right)=R(x, y, z)$ then $\left(\dot{y}_{1}, \dot{y}_{2}, \dot{y_{3}}\right)=$ $-Y\left(y_{1}, y_{2}, y_{3}\right)$.

(b) Since $\dot{y}=4 y z$, the plane $y=0$ is invariant by the flow. The regularized system (5) has equilibrium points given by $(x, 0, z)$ with $4 h+2 x^{2}-x^{4}+2 z^{2}=$ 0 . Applying Lemma 6 , the extension of this curve to the boundary of the Poincar ball is obtained by solving the system

$$
\omega^{4}\left(4 h+2\left(\frac{x}{\omega}\right)^{2}-\left(\frac{x}{\omega}\right)^{4}+2\left(\frac{z}{\omega}\right)^{2}\right)=0, \quad \omega=0 .
$$

It means that the boundary at infinity of this curve is the union of the north and south hemispheres $(0,0, \pm 1)$.

(c) The linearization of $Y$ at $(x, 0, z)$ has the matrix

$$
\left(\begin{array}{ccc}
0 & 0 & 0 \\
0 & 4 z & 0 \\
4 x-4 x^{3} & 0 & 4 z
\end{array}\right) .
$$

It is immediate that the eigenvalues are 0 and $4 z$.

(d) Since $\dot{x}>0$ for $y \neq 0$ it is impossible to have a periodic orbtit.

(e) If $p \in \mathcal{A}$ then $\alpha(p)$ and $\omega(p)$ are contained in $\partial \mathcal{A}$. In fact, since $\varphi(t, p) \in \mathbb{D}^{3}$, and $\mathbb{D}^{3}$ is bounded $\alpha(p)$ and $\omega(p)$ are not empty. Moreover, since $x^{\prime}>0$ in $\mathcal{A}, y^{\prime}>0$ in $\mathbb{D}_{++}^{3}$, and $y^{\prime}<0$ in $\mathbb{D}_{+-}^{3}$, it follows that $\alpha(p), \omega(p) \subset \partial \mathcal{A}$. Due to the fact that $\partial \mathcal{A}$ is invariant, the PoincarBendixson Theorem can be applied. Since there are neither periodic orbits, nor graphics on $\partial \mathcal{A}$ (see Theorem 2) we conclude that all the $\alpha(p)$ and $\omega(p)$ are formed by equilibrium points.

(f) It is a direct consequence of the sign of the eigenvalues of the linearization of $Y$ at $(x, 0, z)$, and the signs of $\dot{x}, \dot{y}$ and $\dot{z}$ in $\mathcal{A}$. 
(g) Statement (c) implies that all equilibrium points in $\mathcal{S}_{h} \cap\{-1<z<$ $1, z \neq 0\}$ are normally hyperbolic. Thus the invariant manifold theory can be applied, see [3].

\section{The $\alpha$ - And $\omega$-Limit Sets of System (5) solutions}

Proof of Theorem 4. (a) Since $\partial \mathcal{A}$ is an invariant set and the phase portrait of the differential system (5) is sketched in figures 1 and 2, the item (a) is proved.

(b) The sign of $\dot{z}$ determines the region where the flow goes up $(\dot{z}>0)$, and the region where the flow goes down $(\dot{z}<0)$. So the surface $\mathcal{S}_{h q}$ is the boundary separating $\mathcal{Z}^{+}$and $\mathcal{Z}^{-}$. The openness follows of the continuity of $\dot{z}$. If the whole orbit passing through $p$ is contained in $\mathcal{Z}^{+}$, then Theorem 3 guarantees that $\omega(p)$ is contained in the set of equilibrium points on $\partial \mathcal{A}$. Moreover, since $\dot{z}>0$ through the orbit, $\omega(p)$ is contained in $L_{N}$. Analogously, if the whole orbit passing through $p$ is contained in $\mathcal{Z}^{-}$, then $\dot{z}<0$ implies the statements.

(c) The boundary of the surface $\dot{z}=4 h+2 x^{2}-x^{4}-2 q y^{2}+2 z^{2}=0$ at infinity is the great circle $x=0$. In fact, according Lemma 6 it follows solving the system

$$
\omega^{4}\left(4 h+2\left(\frac{x}{\omega}\right)^{2}-\left(\frac{x}{\omega}\right)^{4}-2 q\left(\frac{y}{\omega}\right)^{2}+2\left(\frac{z}{\omega}\right)^{2}\right)=0, \quad \omega=0 .
$$

The region on the Poincar ball where $\dot{z}>0$ (the inner one) ends at the circle $\{x=0\}$ of the boundary of the Poincar ball.

In short, we have the following informations about the equilibrium points on $\partial \mathcal{A}$ :

(a) $\mathcal{S}_{h} \cap\{z \geq 0\}$ : contains the $\alpha$ and $\omega$-limit sets of its own points; contains the $\alpha$-limit of any point $p$ at $\mathcal{A}$ which has the whole orbit passing through $p$ contained in the region where $z^{\prime}<0$; and depending on the parameter $h$, contains the $\alpha$ - or the $\omega$ - limit of some orbits on the plane $y=0$ (see figure 2).

(b) North hemisphere $(0,0,1)$ : It is the $\alpha$ and $\omega$-limit of itself; it is $\alpha$ limit of any regular point on $\mathbb{S}^{2}$ and it is the $\omega$ - limit of some orbits on the plane $y=0$ (see figure 2).

(c) Curve $L_{N}$ : contains the $\alpha$ and $\omega$-limit sets of its own points; contains the $\omega$-limit of any point at $\mathcal{A}$ which has the whole orbit passing through it contained in the region where $z^{\prime}>0$.

(d) $\mathcal{S}_{h} \cap\{z \leq 0\}$ : contains the $\alpha$ and $\omega$-limit sets of its own points; contains the $\omega$-limit of any point $p$ at $\mathcal{A}$ which has the whole orbit passing through $p$ contained in the region where $z^{\prime}<0$ and depending on the parameter $h$, contains the $\alpha$ - or the $\omega$ - limit of some orbits on the plane $y=0$ (see figure 2). 
(e) Curve $L_{S}$ : contains the $\alpha$ and $\omega$-limit sets of its own points.

(f) South hemisphere $(0,0,1)$ : It is the $\alpha$ and $\omega$-limit of itself; it is the $\omega$-limit of any regular point on $\mathbb{S}^{2}$ and it is the $\alpha$ - limit of some orbits on the plane $y=0$ (see figure 2).

\section{Conclusions}

We describe the global dynamics of a polynomial differential system in $\mathbb{R}^{4}$ which corresponds to the stationary solutions of the EFK-equation. We find a first integral and thus we reduce our analysis to a family of polynomial differential systems in $\mathbb{R}^{3}$. We provide the global phase portraits of these systems in the Poincaré ball. Moreover we characterize all the $\alpha-$ and $\omega-$ limit sets of all orbits of this system.

\section{REFERENCES}

[1] D. Bonheure, Multitransition kinks and pulses for fourth order equations with a bistable nonlinearity, Ann. I. H. Poincar 21 (2004), 319-340.

[2] A. Cima and J. Llibre, Bounded polynomial vector fields, Trans. Amer. Math. Soc. 318 (1990), 557-579.

[3] M. W. Hirsch, C. C. Pugh and M. Shub, Invariant manifolds, Bull. Amer. Math. Soc. 76 (1970), 1015-1019.

[4] J. Llibre, M. Messias and P. R. da Silva, On the global dynamics of the Rabinovich system, J. Phys. A: Math. Theor. 41(27) (2008), 275210 (21pp).

[5] J. Llibre, M. Messias and P. R. da Silva, Global dynamics of the Lorenz system with invariant algebraic surface, Int. J. Bifur. Chaos Appl. Sci. Engrg. 20 (2010), 31373155.

[6] J. Llibre, J. Sotomayor and M. Zhitomirskii, Impasse bifurcations of constrained systems Differential Equations and Dynamical Systems, Fields Institute Communications, Amer. Mat. Soc. A(31)(2002),235-255.

[7] L.A. Peletier and W.C. Troy, Spatial Patterns, Higher Order Models in Physics and Mechanics, Brikhuser, 2001.

[8] L.A. Peletier and W.C. Troy, Spatial patterns described by the extended FisherKolmogorov (EFK) equation: kinks, Differential and Integral Equations 8 (1995), 1279-1304.

[9] J. Sotomayor and M. Zhitomirskii, Impasse Singularities of Differential Systems of the Form $A(x) x^{\prime}=F(x)$, J. Diff. Equations 169 (2001), 567-587.

${ }^{1}$ Departament de Matemàtiques, Universitat Autònoma de Barcelona, 08193 Bellaterra, Barcelona, Catalonia, Spain.

2 Departamento de Matemática Estatística e Computação - FCT-Unesp, Rua Roberto Simonsen, 305, CEP 19060-900 P. Prudente, São Paulo, Brazil.

3 Departamento de Matemática - IBIlCe-Unesp, Rua C. Colombo, 2265, CEP 15054-000 S. J. Rio Preto, São Paulo, Brazil.

E-mail address: jllibre@mat.uab.cat

E-mail address: marcelo@fct.unesp.br

E-mail address: prs@ibilce.unesp.br 